\title{
Regulation of Neurospora crassa Glutamine Synthetase by the Carbon and Nitrogen Source
}

\author{
By YOLANDA MORA, OFELIA CHÁVEZ AND JAIME MORA* \\ Departamento de Biología Molecular, Instituto de Investigaciones Biomédicas, Universidad \\ Nacional Autónoma de México, Apdo Postal 70228, México 20, D.F., México
}

(Received 25 September 1979)

The effect of the carbon and nitrogen source on the regulation of glutamine synthetase (GS)
has been studied by omitting one or both from mycelium which had been grown in sucrose/
glutamate medium, a condition in which GS is fully induced in its octameric form. The
activity and antigen concentration of GS decreased when mycelium was deprived of the
carbon source. Even more enzyme was degraded when glutamate was replaced by glutamine
and an oligomeric form of GS lower than the octamer was also observed. When the carbon
source was restored, some of the enzyme was resynthesized during incubation with gluta-
mate but not with glutamine. In both cases the final predominant oligomeric form of GS
was the tetramer. When the carbon and nitrogen sources were both omitted from the
medium, degradation of GS again occurred and the restoration of both sources did not result
in an increase in GS: no change in the oligomeric state of GS was observed during these
shifts. The deprivation of the nitrogen source resulted in the replacement of the octamer by
the highly active tetrameric form of GS. These changes were not reversed when either the
carbon or the nitrogen source was again supplied.

\section{INTRODUCTION}

Previous work from this laboratory has indicated that the glutamine synthetase of Neurospora crassa is regulated by the nitrogen source present in the medium. A higher activity was found in the presence of glutamate than with $\mathrm{NH}_{4}{ }^{+}$or glutamine. Immunological titration and sucrose gradient sedimentation of the enzyme established that, under all these conditions, enzyme activity corresponded to enzyme concentration and that the octamer was the predominant oligomeric form (Vichido et al., 1978). Subsequently, it was demonstrated that the differences in enzyme concentration corresponded to modifications in the de novo synthesis of glutamine synthetase (Quinto et al., 1977) and in the concentration of the mRNA specific for this enzyme (Sanchez et al., 1978).

In contrast, both glutamine synthetase and glutamate dehydrogenase in Candida utilis were degraded in the absence of a carbon source (Ferguson \& Sims, $1974 a, b$; Hemmings, 1978). In Aspergillus nidulans the activity of the latter enzyme was also rapidly lost from cultures starved for a carbon source (Hynes, 1974).

The effect of the nitrogen and carbon sources on the regulation of glutamine synthetase has previously been studied in $N$. crassa by regulating its growth by continuously adding the limiting nitrogen or carbon source to the medium. Ammonium limitation led to an elevation of glutamine synthetase; carbon limitation resulted in a decrease in this activity (LimonLason et al., 1977). To determine further the effect of the carbon and nitrogen sources on the regulation of glutamine synthetase in $N$. crassa we have starved the mycelium of its carbon and nitrogen sources and studied the activity, concentration and oligomeric state of glutamine synthetase under these conditions. 


\section{METHODS}

Organism and growth conditions. Neurospora crassa strain 74-A (wild-type) was from the collection of $\mathbf{J}$. Mora. Conidia obtained as previously described (Sanchez et al., 1972) were inoculated into 51 of Vogel's minimal medium $N$ (Vogel, 1964) supplemented with $1.5 \%(\mathrm{w} / \mathrm{v})$ sucrose as carbon source, but with $\mathrm{NH}_{4} \mathrm{NO}_{3}$ substituted by $5 \mathrm{~mm}$-glutamate unless otherwise stated; the medium was sparged with moist air at $25^{\circ} \mathrm{C}$ for 10 to $12 \mathrm{~h}$. Under these conditions the mycelium was growing exponentially with a 3 to $4 \mathrm{~h}$ doubling time and glutamine synthetase was fully induced (Vichido et al., 1978). Mycelium obtained as above was then transferred to minimal medium (MM) with: (i) 5 mM-glutamate as the sole carbon and nitrogen source; (ii) 5 mM-glutamine as the sole carbon and nitrogen source; (iii) neither sucrose nor a nitrogen source; (iv) $1.5 \%$ $(\mathrm{w} / \mathrm{v})$ sucrose without a nitrogen source. Unless otherwise stated, the carbon or nitrogen source omitted was restored after 2 to $5 \mathrm{~h}$.

Glutamine synthetase activity [L-glutamate:ammonia ligase (ADP-forming); EC 6.3.1.2]. Mycelium was separated from the culture medium by filtration through Whatman no. 41 paper, washed with water, then acetone and finally dried under vacuum. All subsequent steps were performed at $4{ }^{\circ} \mathrm{C}$. Acetone powders were ground with dry ice and homogenized with 10 vol. extraction buffer ( 5 mm- $\mathrm{KH}_{2} \mathrm{PO}_{4}, 50 \mathrm{~mm}_{-} \mathrm{K}_{2} \mathrm{SO}_{4}$, $0.5 \mathrm{~mm}$-EDTA, $\mathrm{pH} 7 \cdot 2$ ). The preparation was centrifuged for $20 \mathrm{~min}$ at $12000 \mathrm{~g}$. Both transferase and synthetase activities of the supernatant, which contained approximately $80 \%$ of the original activity, were measured as described by Vichido et al. (1978). The specific activity was expressed as $\mu$ mol $\gamma$-glutamyl hydroxamate formed at $30^{\circ} \mathrm{C} \mathrm{min}^{-1}$ (mg protein) $)^{-1}$. Similar results were obtained when the specific activity of transferase was also determined by measuring the enzyme in both the supernatant and the pellet and dividing the total units by the protein content of the culture.

Sucrose gradient sedimentation. Samples $(0 \cdot 3 \mathrm{ml})$ of the cell-free extracts were layered on a 5 to $20 \%(\mathrm{w} / \mathrm{v})$ continuous sucrose gradient in extraction buffer and centrifuged for $12 \mathrm{~h}$ at $4{ }^{\circ} \mathrm{C}$ in a Spinco L5-75 ultracentrifuge at $40000 \mathrm{rev} . \mathrm{min}^{-1}$ in the SW40 rotor. After centrifugation, $0.3 \mathrm{ml}$ fractions were collected from the top of the tube and their glutamine synthetase activity (transferase) was measured. The molecular weight was calculated as described previously using globular proteins as markers (Palacios, 1976).

Preparation of anti-glutamine synthetase antibodies. Neurospora crassa glutamine synthetase was purified to homogeneity by chromatography on DEAE-cellulose, followed by affinity chromatography on anthranilatebound Sepharose (Palacios, 1976). Goats were immunized and the serum was fractionated with $\left(\mathrm{NH}_{4}\right)_{2} \mathrm{SO}_{4}$ to obtain the total $\gamma$-globulin fraction.

Quantitative immunoelectrophoresis. Rocket immunoelectrophoresis was carried out as described by Weeke (1973) using a $1 \%(\mathrm{w} / \mathrm{v})$ agarose gel containing $1 \%(\mathrm{v} / \mathrm{v})$ anti-glutamine synthetase $\gamma$-globulin fraction. The buffer used was 25 mM-sodium barbital $\mathrm{pH} 8.6$, containing $0.025 \%$ sodium azide. The gel was run for $18 \mathrm{~h}$ at $5 \mathrm{~V} \mathrm{~cm}^{-1}$. After electrophoresis the gel was washed extensively, dried and stained with Coomassie Brilliant Blue R-250. Antigen concentrations were calculated by comparing the areas under the immunoprecipitates with those of standards, as described by Sanchez et al. (1979).

Determination of glutamic acid and glutamine. Samples for glutamic acid and glutamine analysis were prepared by homogenizing mycelium with $80 \%(\mathrm{v} / \mathrm{v})$ ethanol. The homogenates were boiled for $10 \mathrm{~min}$, cooled and filtered through membrane filters (Millipore, type RA, 1.2 $\mu \mathrm{m}$ ). The filtrates containing the amino acids were lyophilized and the samples were resuspended in deionized water. The amino acids were separated using an Aminco (Silver Springs, Md, U.S.A.) amino acid analyser and quantified in an Aminco Ratio Fluorometer after coupling with $o$-phthaldialdehyde. Under these conditions more than $80 \%$ of the glutamic acid and glutamine were recovered.

Determination of 2-oxoglutaric acid. 2-Oxoglutaric acid was assayed according to Bergmeyer \& Bernt (1965) after resuspending the washed mycelium in $0.6 \mathrm{M}-\mathrm{HClO}_{4}$ and neutralizing the supernatant with $\mathrm{K}_{3} \mathrm{PO}_{4}$.

Ammonium determination. Samples $(10 \mathrm{ml})$ of medium were collected by filtration through membrane filters (Millipore, type HA, $0.45 \mu \mathrm{m}$ ) and, after addition of $0.1 \mathrm{ml} 10 \mathrm{M}-\mathrm{NaOH}$, ammonium was measured in an Orion electrode (Cambridge, Mass., U.S.A.) with a membrane specific for this compound. No interference by glutamine was detected if assays were completed within $10 \mathrm{~min}$ of the addition of $\mathrm{NaOH}$.

Protein determination. Samples of mycelium were collected by filtration through membrane filters (Millipore, type HA, $0.45 \mu \mathrm{m}$ ), washed with 2 vol. distilled water and added to $2 \mathrm{ml} 5 \%(\mathrm{w} / \mathrm{v})$ trichloroacetic acid. After centrifugation at $2000 \mathrm{rev} . \mathrm{min}^{-1}$ the pellet was resuspended in $1.0 \mathrm{M}-\mathrm{NaOH}$. Protein was determined by the method of Lowry using bovine serum albumin as standard.

Chemicals. Amino acids, bovine serum albumin, glutamic acid dehydrogenase, NADPH, 2-oxoglutaric acid, and $o$-phthaldialdehyde were obtained from Sigma. Agarose and immunoelectrophoresis barbital buffer were from Bio-Rad Laboratories. 

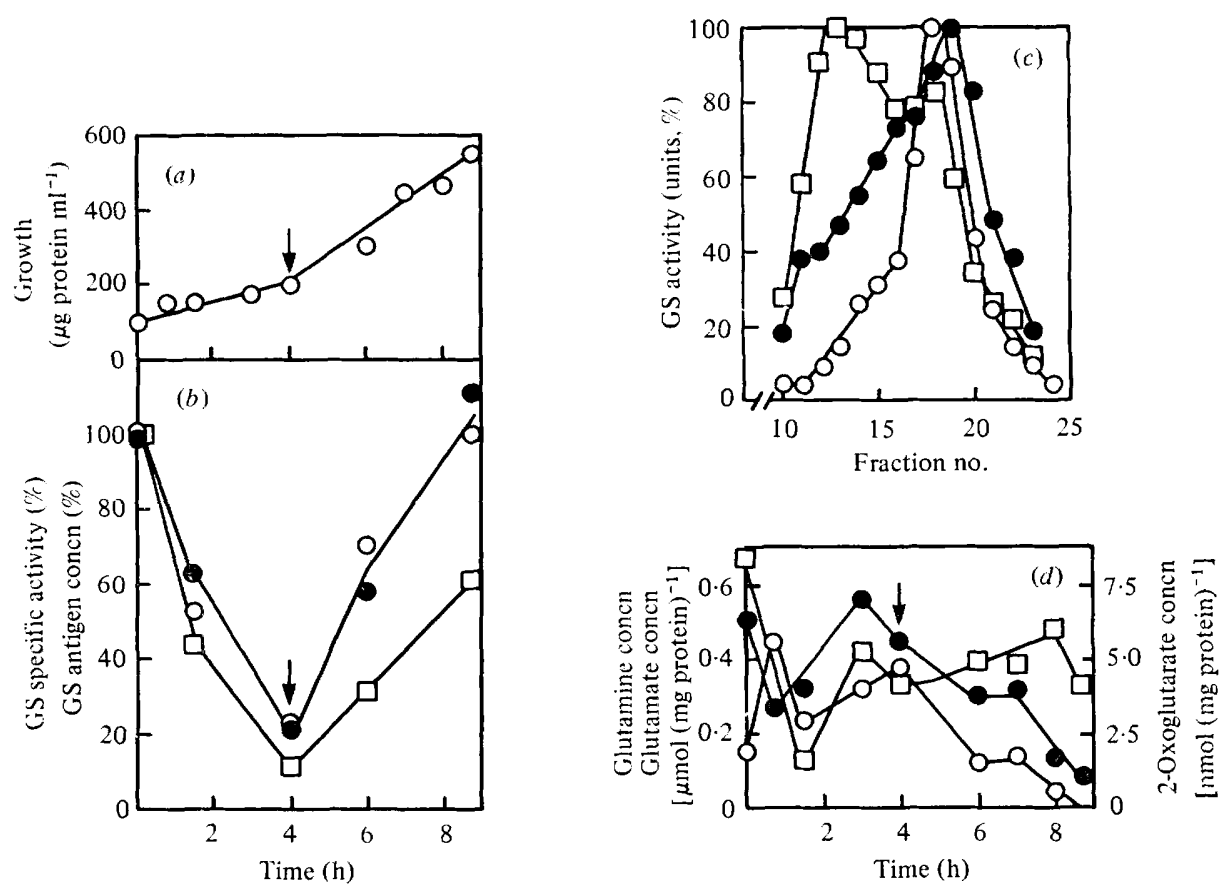

Fig. 1. Growth, glutamine synthetase activity, concentration of GS antigen, amino acid pools and sedimentation of GS from $N$. crassa mycelium after transfer to medium containing glutamate as nitrogen and carbon source and after subsequent addition of sucrose. Neurospora crassa was grown for $10.5 \mathrm{~h}$ in $\mathrm{MM}$ as described in Methods, with $\mathrm{NH}_{4} \mathrm{NO}_{3}$ replaced by glutamate $(5 \mathrm{~mm})$. The mycelium was then filtered, washed with water, and transferred to medium containing glutamate $(5 \mathrm{~mm})$ as the only carbon and nitrogen source; after $4 \mathrm{~h}$ (arrow), $1.5 \%(\mathrm{w} / \mathrm{v})$ sucrose was added. (a) Growth. (b) GS specific activity measured by the synthetase $(\bigcirc)$ or transferase $(O)$ assays and expressed as a percentage of the activity at zero time $(0.061$ units of synthetase and 0.27 units of transferase); antigen concentration ( $\square$ ) expressed as a percentage of the concentration at zero time. (c) Sedimentation of GS (see Methods) at zero time ( $\bigcirc)$, after $4 \mathrm{~h}$ in glutamate without sucrose ( $\bullet$ ), and at $4.75 \mathrm{~h}$ after the addition of sucrose $([]) .(d)$ Metabolite pools: glutamine $(O)$, glutamate $(\bullet)$ and 2-oxoglutarate ([ $[$ ).

\section{RESULTS}

\section{Deprivation of the carbon source in the presence of glutamate}

Mycelium transferred to a medium without sucrose grew slowly with glutamate as nitrogen and carbon source. When sucrose was supplied, the mycelium grew at a rate similar to that before the deprivation (Fig. 1 $a$ ). There was an $80 \%$ decrease in transferase and synthetase activities of glutamine synthetase (GS) after $4 \mathrm{~h}$ of deprivation (Fig. $1 b$ ). The addition of sucrose to starvation medium at this time resulted in a linear increase in both activities to $100 \%$ of the original activity $5 \mathrm{~h}$ later. The change in antigen concentration, estimated by quantitative immunoelectrophoresis, paralleled the enzymic activity during deprivation, indicating that there was a degradation of GS; however, the addition of sucrose only partially restored the original concentration of antigen (Fig. 1b). Most important was the change in the oligomeric state of GS which occurred when the available nutrients were varied. Before deprivation, GS sedimented in a sucrose gradient as an octamer, with a small shoulder corresponding to a lower oligomeric form (Fig. 1c). The shoulder was more extended and prominent after $4 \mathrm{~h}$ of carbon deprivation. The subsequent addition of sucrose to the culture did not prevent the appearance of a tetramer which was relatively more abundant than the octamer (Fig. 1c; Vichido et al., 1978; Limon-Lason et al., 1977).

There was an $80 \%$ decrease in the concentration of 2-oxoglutarate in the mycelium during 

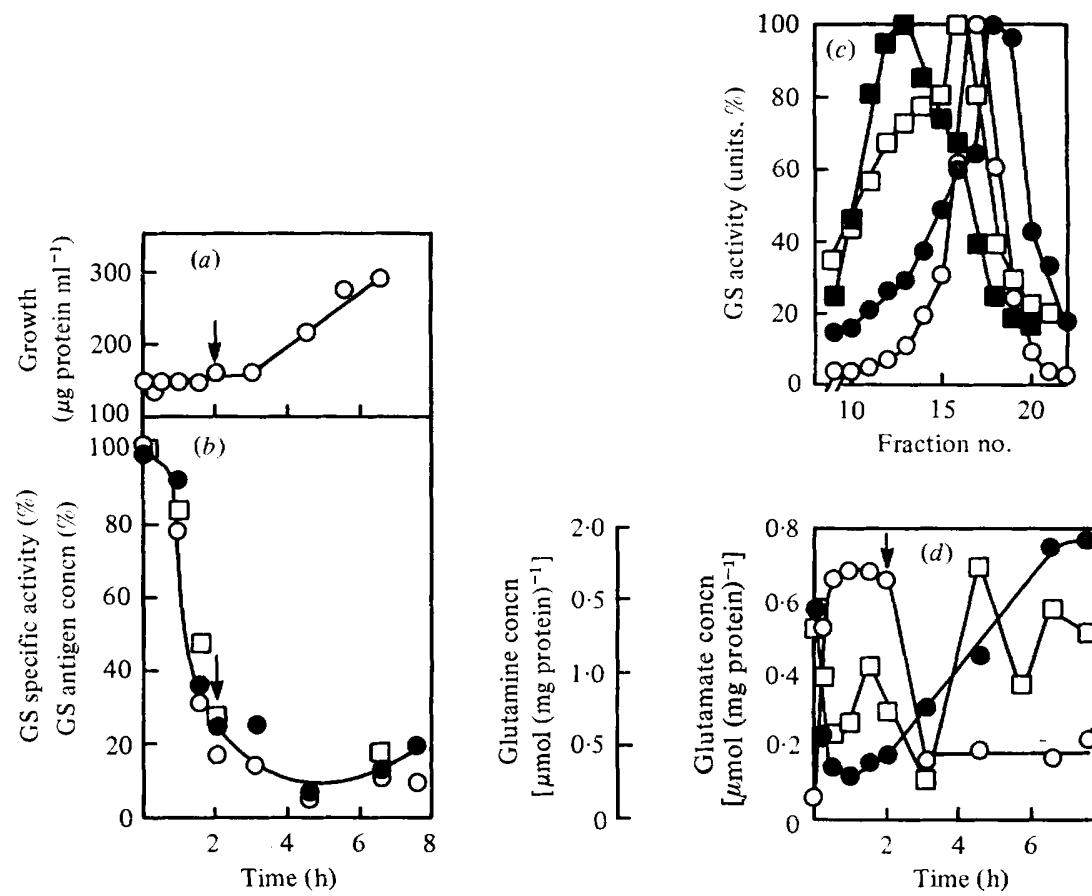

Fig. 2. Growth, glutamine synthetase activity, concentration of GS antigen, amino acid pools and sedimentation of GS from $N$. crassa mycelium after transfer to medium containing glutamine as nitrogen and carbon source and after subsequent addition of sucrose. Neurospora crassa was grown for $11 \mathrm{~h}$ as described in the legend to Fig. 1. The mycelium was then filtered, washed with water, and transferred to medium containing glutamine $(5 \mathrm{~mm})$ as the only carbon and nitrogen source; after $2 \mathrm{~h}$ (arrow), $1.5 \%$ (w/v) sucrose was added. ( $a, b$ and $d$ ) Symbols as in the legend to Fig. 1; in $(b)$, the GS specific activity at zero time was 0.111 units of synthetase and 0.427 units of transferase. (c) Sedimentation of GS after $30 \mathrm{~min}(\bigcirc)$ and $130 \mathrm{~min}(O)$ in glutamine and $1 \mathrm{~h}(\square)$ and $5.5 \mathrm{~h}(\mathbb{\square})$ after the addition of sucrose.

the initial $1.5 \mathrm{~h}$ of carbon deprivation; the concentration subsequently increased to $50 \%$ of the initial value, even after adding sucrose. The concentration of glutamate decreased by $45 \%$ during the first $45 \mathrm{~min}$ of deprivation but returned to the initial value $3 \mathrm{~h}$ later. The glutamine concentration increased threefold during the first $45 \mathrm{~min}$ of deprivation but subsequently decreased (Fig. $1 d$ ). When sucrose was added to the culture, the glutamate and glutamine concentrations decreased considerably (Fig. 1d).

\section{Deprivation of the carbon source in the presence of glutamine}

As glutamine represses the synthesis of GS (Vichido et al., 1978; Quinto et al., 1977), we decided to study the effect of glutamine on the degradation of GS during deprivation of the carbon source.

No growth occurred when mycelium which had been grown with sucrose was incubated with glutamine as the only carbon source. When sucrose was added growth recommenced at a rate lower than that when glutamine was the initial nitrogen source (Fig. $2 a$; Vichido et al., 1978). The activity of GS had decreased by $75 \%$ after $2 \mathrm{~h}$ of carbon deprivation, and on subsequent readdition of sucrose a further decrease in activity occurred (Fig. $2 b$ ). The antigen concentration decreased in parallel with the loss of enzyme activity: the degradation of the enzyme was therefore more rapid during incubation with glutamine than with glutamate (Fig. $1 b, 2 b$ ). The oligomeric state of GS again changed under these conditions to the tetrameric form. During the first $30 \mathrm{~min}$ of carbon deprivation the activity of GS 

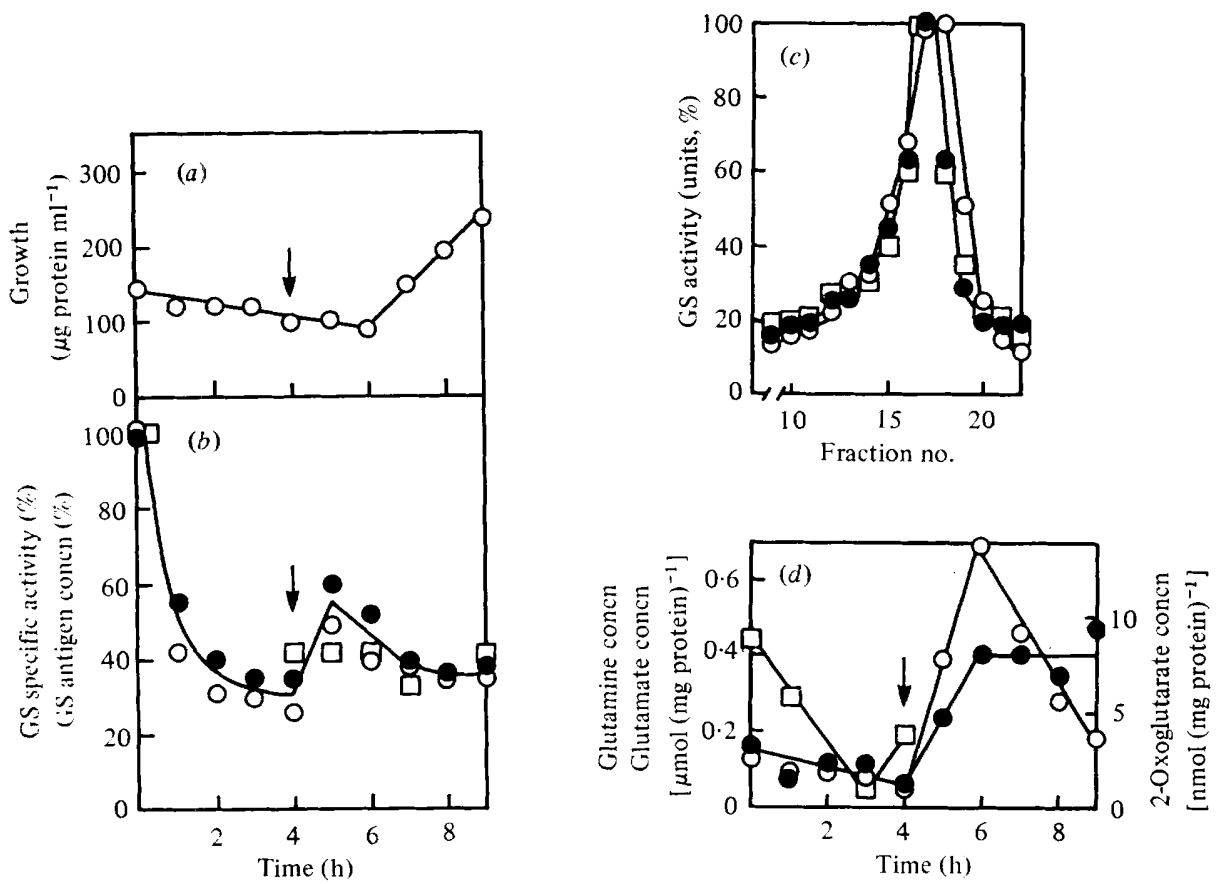

Fig. 3. Growth, glutamine synthetase activity, concentration of GS antigen, amino acid pools and sedimentation of GS from $N$. crassa mycelium after transfer to a medium lacking nitrogen and carbon sources and after the subsequent addition of sucrose and glutamate. Neurospora crassa was grown for $10.5 \mathrm{~h}$ as described in the legend to Fig. 1. The mycelium was then filtered, washed with water and transferred to MM with neither sucrose nor nitrogen source; after $4 \mathrm{~h}$ (arrow), $5 \mathrm{mm-}$ glutamate and $1.5 \%(\mathrm{w} / \mathrm{v})$ sucrose were added. $(a, b$ and $d)$ Symbols as in the legend to Fig. 1 ; in (b), the GS specific activity at zero time was $0 \cdot 108$ units of synthetase and 0.391 units of transferase. (c) Sedimentation of GS $4 \mathrm{~h}$ after deprivation of sucrose and nitrogen $(O)$ and $1 \mathrm{~h}(\mathrm{O})$ and $3 \mathrm{~h}$ (-) after resupplementation with sucrose and glutamate.

sedimented through a sucrose gradient as an octamer and $2 \mathrm{~h}$ later a shoulder appeared due to a lower oligomeric form. At $5 \mathrm{~h}$ after sucrose was restored to the starvation medium, the tetramer was the major species, with a shoulder corresponding to a higher oligomeric form (Fig. 2c). The degradation of GS was associated in this case with a rapid increase in the glutamine pool (10-fold) and a large depletion of the glutamate pool (Fig. $2 d$ ). When sucrose was added to the starvation medium, the glutamine concentration decreased abruptly during the first hour but a high pool was maintained for the following $4.5 \mathrm{~h}$. Glutamate increased to a concentration similar to that found during growth with glutamate and sucrose (Fig. $2 d$ ).

\section{Deprivation of the carbon and nitrogen source}

To test the effect of the absence of a nitrogen source on the degradation of the enzyme, the mycelium was transferred to a medium lacking both glutamate and sucrose. After this shift the protein in the culture was degraded and ammonium was excreted into the medium (Fig. $3 a, 4 a, 4 b$ ). Growth recommenced $2 \mathrm{~h}$ after the starvation medium was supplemented with sucrose and glutamate (Fig. $3 a$ ).

Deprivation of carbon and nitrogen for $2 \mathrm{~h}$ was sufficient to decrease the activity of GS by $60 \%$. No further degradation occurred during the subsequent $2 \mathrm{~h}$. When sucrose and glutamate were added the activity increased a little in the first hour and subsequently decreased to the value at the end of deprivation (Fig. 3 b). There was also a $60 \%$ decrease in the antigen concentration after $4 \mathrm{~h}$ of carbon and nitrogen deprivation, and even after restoration of both sources, the antigen concentration did not change appreciably (Fig. $3 b$ ). As these 

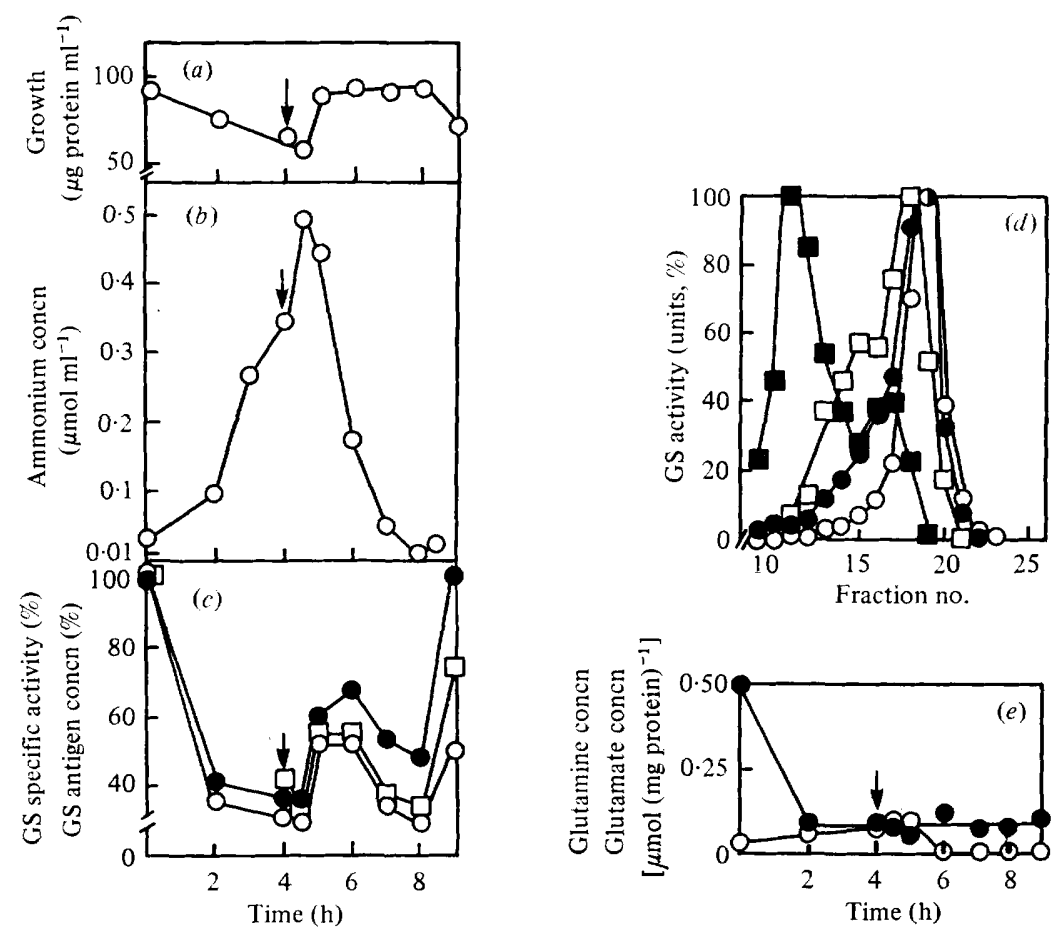

Fig. 4. Growth, excretion of ammonium, glutamine synthetase activity, concentration of GS antigen, amino acid pools and sedimentation of GS from $N$. crassa mycelium after transfer to a medium lacking nitrogen and carbon sources and after subsequent addition of sucrose. Neurospora crassa was grown for $10 \mathrm{~h}$ as described in the legend to Fig. 1. The mycelium was then filtered, washed with water and transferred to MM with neither sucrose nor nitrogen source; after $4 \mathrm{~h}$ (arrow), $1.5 \%(\mathrm{w} / \mathrm{v})$ sucrose alone was added. ( $a, c$ and $e$ ) Symbols as in the legend to Fig. $1(a, b$ and $d)$; in (c) the GS specific activity at zero time was 0.109 units of synthetase and 0.43 units of transferase. (b) Ammonium excreted into the medium. (d) Sedimentation of GS at zero time $(\bigcirc)$, $4 \mathrm{~h}$ after deprivation of carbon and nitrogen (O) and $3 \mathrm{~h}(\square)$ and $5 \mathrm{~h}(\square)$ after the addition of sucrose.

results are from a single experiment there is some uncertainty about the significance of the small increase in GS activity which was not reflected by a parallel increase in antigen concentration. As shown below, the glutamine accumulated in this condition does not necessarily require an elevation of GS. In contrast to the appearance of the tetramer during carbon deprivation, the octamer predominated throughout the period of carbon and nitrogen deprivation (Fig. 3c). During deprivation, mycelial concentrations of glutamate and glutamine remained low and constant. Growth did not start immediately when sucrose and glutamate were supplied to the culture medium and the pools of glutamate and glutamine increased rapidly. As growth started, the pool of glutamine decreased but that of glutamate remained high (Fig. $3 d$ ).

Ferguson \& Sims (1974b) reported that when yeast which had been grown with glucose and glutamate was transferred to a medium lacking both substrates, GS was rapidly inactivated and the enzyme reappeared when glucose was restored. To determine whether the enzyme reappeared in $N$. crassa under the same conditions, only sucrose was added back to the medium after carbon and nitrogen deprivation. A small increase in protein concentration was observed as a result of the utilization of the ammonium ions previously secreted into the medium (Fig. $4 a, b$ ). Only $1 \mathrm{~h}$ after sucrose addition, GS activity had increased but it subsequently declined to that found at the end of deprivation. Although both activities followed the same general pattern of variation, a diminution of the ratio of synthetase to 

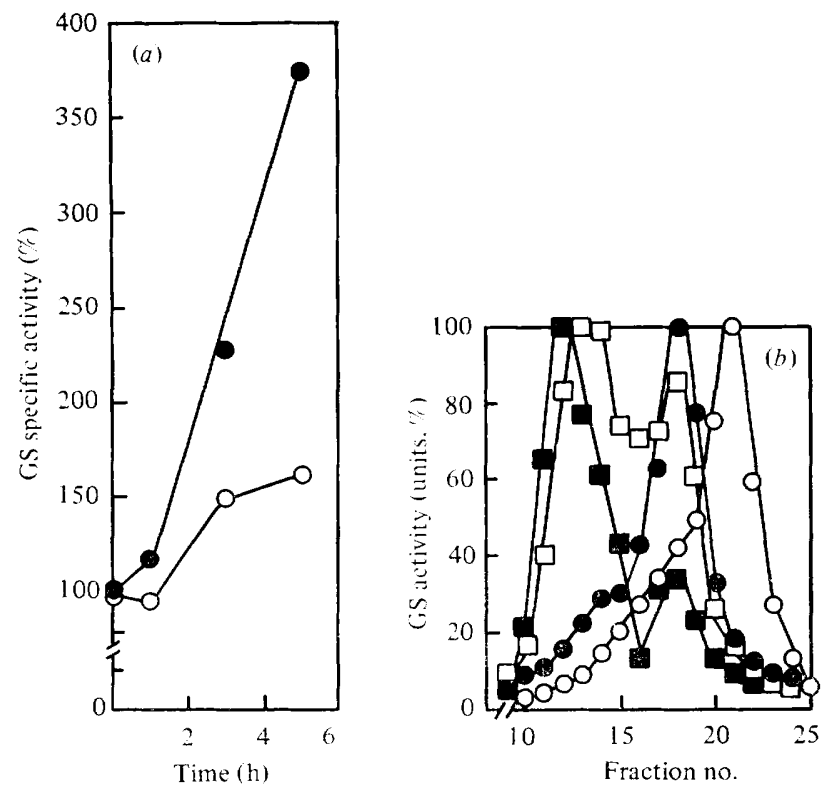

Fig. 5. Glutamine synthetase activity and sedimentation of GS from $N$. crassa mycelium incubated with sucrose in the absence of a nitrogen source. Neurospora crassa was grown for $12 \mathrm{~h}$ in MM with $25 \mathrm{~mm}-\mathrm{NH}_{4} \mathrm{NO}_{3}$ as described in Methods. The mycelium was then filtered, washed with water and transferred to MM with $1.5 \%(\mathrm{w} / \mathrm{v})$ sucrose and no nitrogen source. (a) GS specific activity, symbols as in the legend to Fig. 1; the activity at zero time was 0.021 units of synthetase and 0.096 units of

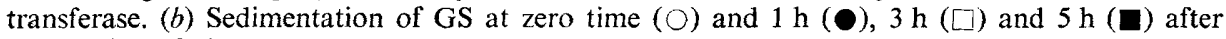
deprivation of nitrogen.

transferase activity was observed. This change of ratio was apparent $5 \mathrm{~h}$ after sucrose addition by which time the transferase activity had increased to the original level (Fig. $4 c$ ). The antigen concentration generally followed the changes in enzyme activity (Fig. $4 c$ ). By $3 \mathrm{~h}$ after restoration of sucrose a shoulder due to tetrameric GS became apparent in the sucrose gradient, and $2 \mathrm{~h}$ later the tetramer was the predominant form (Fig. $4 d$ ). The glutamate pool remained low and constant and glutamine was completely depleted (Fig. $4 e$ ).

\section{Deprivation of the nitrogen source}

The previous results indicated that, in the absence of a nitrogen source, sucrose stimulated a change in GS from the octameric to the tetrameric form. It was important, therefore, to determine whether nitrogen starvation was essential for this modification. Mycelium with a low GS activity (Vichido et al., 1978) was grown for $12 \mathrm{~h}$ in MM and transferred to a nitrogen-free medium containing sucrose. The transferase activity increased far more rapidly than the synthetase (Fig. $5 a$ ) and the predominant oligomeric state of the enzyme after $5 \mathrm{~h}$ was the tetramer (Fig. $5 b$ ), indicating that previous deprivation of carbon and nitrogen was not necessary to promote the appearance of this oligomer. The sequential appearance of oligomeric intermediates between the octamer and the tetramer was observed during the nitrogen deprivation (Fig. $5 b$ ).

With the exception of the experiment in which sucrose and glutamate were restored to the starvation medium, all the others were repeated several times with essentially similar results. 


\section{DISCUSSION}

Mycelium of Neurospora crassa degraded GS when it was deprived of a carbon source. This would explain the low activity of this enzyme in carbon-limited cultures (Limon-Lason et al., 1977). This degradation was enhanced when glutamine was present instead of glutamate (Fig. $1 b, 2 b$ ). It is known that glutamine represses the de novo synthesis of GS by decreasing the concentration of mRNA specific for this enzyme (Quinto et al., 1977; Sanchez et al., 1978). Because the rate of degradation of GS was similar in the presence or absence of glutamate, we suggest that the high content of glutamine under these conditions (Fig. $2 d$ ) has an additional effect of regulating the degradation of this enzyme (Fig. $2 b$ ).

When the mycelium was deprived of carbon and nitrogen, protein was degraded to ammonium and excreted into the medium (Fig. $4 a, b$ ). We propose that the specific degradation of GS is a regulatory mechanism to prevent the synthesis of glutamine and spare carbon skeletons to provide energy. The observations that, in Candida utilis, GS (Ferguson \& Sims, $1974 a, b)$ and the biosynthetic glutamate dehydrogenase (Hemmings, 1978) are degraded in the absence of a carbon source, support this contention.

The appearance of the tetrameric GS was not a necessary condition for degradation to occur (Fig. $3 c, 4 d$ ). In $C$. utilis, the inactivation of GS by glutamine is accompanied by a change from the octameric to the tetrameric form of GS (Ferguson \& Sims, 1974b; Sims et al., 1974a). Previously we have reported that the shift of cultures of $N$. crassa from growth with glutamate to growth with glutamine prevented the synthesis of the octameric form; no tetrameric form of the enzyme was apparent during these studies (Vichido et al., 1978; Quinto et al., 1977). However, nitrogen deprivation in $N$. crassa resulted in the disappearance of octameric GS and the appearance of the tetrameric form. Under similar conditions the GS activity in C. utilis increased only slightly and no information is available about its oligomeric state (Sims et al., 1974b).

Restoration of the carbon source did not generally restore the mycelium to its original metabolic condition. As shown here, the deprivation of carbon or nitrogen triggers a change of the octameric GS to a tetrameric enzyme. We have commented before (Vichido et al., 1978 ) that the changes in C. utilis were freely reversible, but this is not the case in N. crassa. In a filamentous fungus with a vegetative cycle developmental processes are not excluded.

When mycelium which had been incubated with glutamine alone was supplemented with a carbon source, there was a diminution of the glutamine pool, an increase in 2-oxoglutarate to concentrations similar to those before deprivation and an increase in glutamate to concentrations similar to those found when glutamate was the nitrogen source (Fig. $2 d$ ). These results indicate that a carbon source is necessary for the conversion of glutamine to glutamate. We have previously reported that in $N$. crassa glutamine is catabolized to glutamate by the $\omega$-amidase pathway (Espín et al., 1979).

This work was supported in part by CONACyT, Mexico. We are grateful to Dr Rafael Palacios for advice and his critical review of the manuscript.

\section{REFERENCES}

Bergmeyer, H. U. \& Bernt, E. (1965). In Methods in Enzymatic Analysis, pp. 324-327. Edited by H. U. Bergmeyer. New York: Academic Press.

Espín, G., Palacios, R. \& Mora, J. (1979). Glutamine metabolism in nitrogen-starved conidia of Neurospora crassa. Journal of General Microbiology 115, 59-68.

Ferguson, A. R. \& Sims, A. P. (1974a). The regulation of glutamine metabolism in Candida utilis: the role of glutamine in the control of glutamine synthetase. Journal of General Microbiology 80, 159-171.

Ferguson, A. R. \& Sims, A. P. (1974b). The regulation of glutamine metabolism in Candida utilis: the inactivation of glutamine synthetase. Journal of General Microbiology 80, 173-185.

Hemmings, B. A (1978). Evidence for the degradation of nicotinamide adenine dinucleotide 
phosphate-dependent glutamate dehydrogenase of Candida utilis during rapid enzyme inactivation. Journal of Bacteriology 133, 867-877.

HyNES, M. J. (1974). The effects of the carbon source on glutamate dehydrogenase activities in Aspergillus nidulans. Journal of General Microbiology 81, 165-170.

Limon-Lason, J., Lara, M., Resendiz, B. \& Mora, J. (1977). Regulation of glutamine synthetase in fed-batch cultures of Neurospora crassa. Biochemical and Biophysical Research Communications 78, 1234-1240.

Palacios, R. (1976). Neurospora crassa glutamine synthetase. Purification by affinity chromatography and characterization of subunit structure. Journal of Biological Chemistry 251, 4787-4791.

Quinto, C., Mora, J. \& Palacios, R. (1977). Neurospora crassa glutamine synthetase. Role of enzyme synthesis and degradation on the regulation of enzyme concentration during exponential growth. Journal of Biological Chemistry 252, 8724-8727.

Sanchez, S., Martinez, L. \& Mora, J. (1972). Interactions between amino acid transport systems in Neurospora crassa. Journal of Bacteriology 112, 276-284.

Sanchez, F., Campomanes, M., Quinto, C., Hansberg, W., Mora, J. \& Palacios, R. (1978). Nitrogen source regulates glutamine synthetase
mRNA levels in Neurospora crassa. Journal of Bacteriology 136, 880-885.

Sanchez, F., Davila, G., Mora, J. \& Palacios, R. (1979). Immunochemical characterization of glutamine synthetase from Neurospora crassa glutamine auxotrophs. Journal of Bacteriology 139, 537-543.

Sims, A. P., Toone, J. \& Box, V. (1974a). The regulation of glutamine synthesis in the food yeast Candida utilis: the purification and subunit structure of glutamine synthetase and aspects of enzyme deactivation. Journal of General Microbiology 80, 485-499.

Sims, A. P., Toone, J. \& Box, V. (1974b). The regulation of glutamine metabolism in Candida utilis: mechanism of control of glutamine synthetase. Journal of General Microbiology 84, 149-162.

Vichido, I., Mora, Y., Quinto, C., Palacios, R. \& MoRA, J. (1978). Nitrogen regulation of glutamine synthetase in Neurospora crassa. Journal of General Microbiology 106, 251-259.

VoGEL, H. J. (1964). Distribution of lysine pathways among fungi: evolutionary implications. American Naturalist 98, 435-446.

WEEKE, B. (1973). Rocket immunoelectrophoresis. In $A$ Manual of Quantitative Immunoelectrophoresis, pp. 37-46. Edited by N. V. Axelsen, J. Krøll \& B. Weeke. Oslo: Universitetsforlaget. 